\title{
PROCESSO DE ALTA HOSPITALAR DA CRIANÇA: PERCEPÇÕES DE ENFERMEIROS ACERCA DOS LIMITES E DAS POTENCIALIDADES DE SUA PRÁTICA PARA A ATENÇÃO INTEGRAL ${ }^{1}$
}

\author{
Raquel Vicentina Gomes de Oliveira Silva², Flávia Regina Souza Ramos ${ }^{3}$
}

\footnotetext{
${ }^{1}$ Parte da dissertação - O trabalho de enfermagem na articulação entre atenção hospitalar e atenção básica na pós-alta da criança: subsídios para a integralidade, apresentada ao Programa de Pós-Graduação em Enfermagem (PEN) da Universidade Federal de Santa Catarina (UFSC), 2009.

${ }^{2}$ Especialista em Saúde da Família. Enfermeira do Hospital Universitário da UFSC. Santa Catarina, Brasil. E-mail: raquelenfer@ yahoo.com.br

${ }^{3}$ Doutora em Enfermagem. Professora Adjunto do Departamento e da PEN/UFSC. Pesquisadora do CNPq. Santa Catarina, Brasil. E-mail: flaviar@ccs.ufsc.br
}

RESUMO: A integralidade é um dos pilares de sustentação do Sistema de Saúde Brasileiro e a busca por sua efetivação deve ocorrer nos diferentes níveis e serviços de saúde, especialmente na capacidade de articulação entre eles. Este estudo objetivou identificar as percepções de enfermeiros acerca dos limites e das potencialidades do seu trabalho para a integralidade do cuidado na articulação entre atenção hospitalar e básica no processo de alta hospitalar da criança. A pesquisa qualitativa, exploratória e descritiva contou com a participação de 13 enfermeiras, sendo sete da atenção hospitalar e seis da atenção básica, de um município da região Sul do Brasil. Como principal limite surgiu a ausência de comunicação entre as duas esferas e como potencialidade, a alta cobertura de equipes de Estratégia Saúde da Família. Concluiu-se, na perspectiva da integralidade, a necessidade de um repensar dos profissionais envolvidos nessa prática, a fim de modificar o atual cenário.

DESCRITORES: Assistência integral à saúde. Cuidados de enfermagem. Alta do paciente.

\section{THE PROCESS OF RELEASING A CHILD FROM THE HOSPITAL: NURSING PERCEPTIONS CONCERNING LIMITS AND POTENTIALITIES OF ITS PART IN INTEGRAL CARE}

\begin{abstract}
Integrality is one of the pillars of the Brazilian National Health Care System (Sistema Único de Saúde) and the search for its effectuation must occur within the different levels and services of health care, especially concerning the capacity for articulation among them. This study aims to identify nursing perceptions about the limits and possibilities of their work towards comprehensive, hospital, and basic care in the hospital release process involving a child. This descriptive, qualitative research counted on the participation of 13 nurses, seven from the hospital and six from primary care in a municipality in southern Brazil. The absence of communication between the two spheres emerged as the principle limit, while the high coverage of the Family Health Strategy teams emerged as the principle potential. Under the perspective of integrality, we conclude there is a need for rethinking among professionals involved in this practice in order to modify the current scenario.
\end{abstract}

DESCRIPTORS: Comprehensive health care. Nursing care. Patient discharge.

\section{LA PRÁCTICA INTEGRAL DEL ENFERMERO EN EL PROCESO DEL ALTA HOSPITALARIA DEL NIÑO: LÍMITES Y POTENCIALIDADES}

\begin{abstract}
RESUMEN: La integralidad es uno de los pilares del Sistema Brasileño de Salud y la búsqueda de su aplicación debe producirse en diferentes niveles y servicios de salud y, especialmente, en la capacidad de articularlos. El objetivo del estudio fue identificar las percepciones de los enfermeros sobre los límites y las posibilidades de su trabajo para la integralidad del cuidado, en lo que se refiere a la relación: atención hospitalaria y atención básica en el proceso del alta hospitalaria del niño. Se trata de una investigación cualitativa de tipo descriptivo, con la participación de 13 enfermeras: siete de la atención hospitalaria y seis de la atención básica, de un municipio de la región sur del Brasil. Los resultaron mostraron como principal límite la falta de comunicación entre las dos esferas, y como potencialidades, la amplia cobertura de los equipos de la Estrategia de Salud Familiar. Se concluye que desde la perspectiva de la integralidad, es necesario que los profesionales involucrados en esa práctica repiensen su actuación, con el objetivo de modificar la actual situación.
\end{abstract}

DESCRIPTORES: Asistencia integral en salud. Atención de enfermería. Alta del paciente. 


\section{INTRODUÇÃO}

O Sistema Único de Saúde (SUS) é considerado um sistema complexo, pois tem a responsabilidade de articular e coordenar ações promocionais e de prevenção com as de cura e reabilitação. ${ }^{1}$ Conforme a Lei Orgânica n ${ }^{\circ} 8.080$, de 19 de setembro de 1990, a construção do SUS é norteada por alguns princípios e diretrizes, entre os quais a integralidade. ${ }^{2}$ Esta é uma das diretrizes mestras da reforma do Sistema de Saúde Brasileiro, portanto, deve ser ponto de reflexão o seu alcance, bem como seu limite e efetivação na prática dos serviços em saúde, levando em consideração a eficiência e eficácia do SUS. ${ }^{3}$

A integralidade é um dos pilares a sustentar a criação do SUS. ${ }^{4}$ No setor saúde, é vista como um termo novo e vem designando várias dimensões e sentidos. Seu caráter polissêmico é fértil, e por isso não se pretende dar qualquer definição unívoca para abraçar os pelo menos três grandes conjuntos de seus sentidos: para designar atributos e valores de algumas práticas de saúde, alguns modos de organizar os serviços de saúde e de algumas políticas de saúde. ${ }^{5}$

Nesse contexto, entende-se que a integralidade deve ser trabalhada em suas várias dimensões, para que seja alcançada em sua forma mais ampla. Assim, deve ser percebida em uma dimensão sistêmica, em relação a uma atenção contínua, através de ações articuladas em rede, que promovam a coordenação do cuidado entre os diversos serviços de saúde. ${ }^{6}$ Em outra dimensão, mas interligada à primeira, está a integralidade em sua dimensão de práticas de saúde, quando cada profissional deve buscar a construção de práticas de atenção integral à saúde no seu dia-a-dia, tendo em vista uma assistência qualificada, que veja o indivíduo como um todo, preservando seus direitos e valores. ${ }^{3}$

No âmbito hospitalar, a integralidade da atenção recebida resultará de como está articulada a prática dos trabalhadores. O hospital contribuiria para a integralidade do cuidado quando no sistema de saúde fosse referência e, após atendimento, fizesse uma adequada contrarreferência. Entretanto, a implementação efetiva desses "circuitos base-topo e topo-base" nem sempre é muito bemsucedida na prática. ${ }^{7}$

Por outro lado, a atenção básica é um componente fundamental dos sistemas de saúde, tendo como papel a prestação direta dos serviços e providenciando ou coordenando a prestação destes em outros locais. ${ }^{8}$
Reporta-se que, especificamente, no processo de alta hospitalar da criança, cabe considerar a importância da atuação profissional, para a continuidade e integralidade do cuidado. A alta da criança hospitalizada é vista como um processo que se consolida no momento final da internação hospitalar e continua no período pós-hospitalar. ${ }^{9}$ É, também, um momento privilegiado para concretizar o cuidado integral. O cuidado integral em saúde acontece quando existe uma combinação generosa e flexível de tecnologias duras, leve-duras e leves ${ }^{7}$, de modo a contemplar a complexidade das necessidades e do objeto da ação em saúde.

A criança e sua hospitalização têm sido tema de discussão da enfermagem. Nos últimos anos, estudos denotam uma preocupação com a dimensão cuidadora da enfermagem, à criança e à família, exercida durante a permanência da criança no hospital ${ }^{10-13}$ e/ou em Unidades de Saúde na Atenção Básica. ${ }^{14}$ Fica evidente, também, o foco sobre a reorganização do processo de trabalho para a qualidade do cuidado. ${ }^{15-17}$ Entretanto, a questão da lacuna na integralidade da assistência - bem como dos limites e das potencialidades da atuação no processo de alta hospitalar da criança - necessita ser aprofundada, para que mudanças ocorram no processo de trabalho de enfermagem. Faz-se necessário um planejamento para a alta hospitalar. ${ }^{18}$

Entre os envolvidos no processo de alta, encontra-se o enfermeiro, sendo atribuição deste profissional, seja da atenção hospitalar ou da atenção básica, promover a continuidade da assistência do hospital ao domicílio; uma assistência integrada e comprometida com a saúde. Considerando a integralidade do cuidado, o enfermeiro deve favorecer a articulação da atenção hospitalar e básica, contribuindo para a construção deste princípio. Entretanto, na busca por um agir integral, despontam alguns limites e potencialidades que impedem e/ou contribuem para tal construção. Com tal ênfase, não se pretende negligenciar a responsabilidade dos outros profissionais de saúde ${ }^{4}$ e da própria família para a efetivação do cuidado integral.

Este artigo relata parte dos resultados de uma dissertação de mestrado intitulada "O trabalho de enfermagem na articulação entre atenção hospitalar e atenção básica na pós-alta da criança subsídios para a integralidade". Tem como objetivo identificar limites e potencialidades do trabalho do enfermeiro para a integralidade do cuidado na articulação entre atenção básica e hospitalar no processo de alta hospitalar da criança/família. 


\section{MÉTODO}

Trata-se de um estudo descritivo-exploratório, com uma abordagem qualitativa, desenvolvido em uma Unidade de Internação Pediátrica (UIP) de um hospital público, de ensino, e em cinco Unidades Locais de Saúde (ULSs) de um município da região Sul do Brasil. A escolha desse cenário se deu pelo fato de ser o município em que a autora reside e o local onde atua. As ULSs e suas enfermeiras foram selecionadas através de um sorteio prévio realizado pela própria pesquisadora. Uma enfermeira da atenção básica, responsável pela Saúde da Criança, foi intencionalmente selecionada.

Os participantes do estudo foram sete enfermeiras atuantes em uma UIP e seis enfermeiros atuantes na atenção básica/Estratégia de Saúde da Família.

Como instrumento de coleta de dados, foi utilizada a entrevista semiestruturada. As entrevistas foram agendadas e realizadas pela pesquisadora, no próprio ambiente de trabalho dos participantes, nos meses de dezembro de 2008 e janeiro de 2009, após consentimento dos participantes, segundo Resolução 196/96 do Conselho Nacional de Saúde. Todas foram registradas por gravação de áudio e posteriormente transcritas. Observando os aspectos éticos e legais, foi respeitada a liberdade de participação dos enfermeiros e seu anonimato foi garantido com o uso de códigos para identificar suas falas (E1 ESF, E2 ESF, E1 UIP, E2 UIP), de acordo com a ordem cronológica de realização das entrevistas e do local de trabalho - ESF e UIP. Obteve-se a autorização junto aos locais onde ocorreu a pesquisa e a aprovação do Comitê de Ética e Pesquisa com Seres Humanos da Universidade Federal de Santa Catarina (certificado 291).

A partir das transcrições das entrevistas, a análise dos dados foi realizada com os seguintes passos: leitura, releitura e análise individual das entrevistas; seleção e identificação das unidades de significado; organização das unidades de significado por semelhança de conteúdo; e constituição das categorias. ${ }^{18}$ Para ajudar na análise, utilizamos um software de análise qualitativa, o ATLAS. ti. A discussão dos dados deu-se pela articulação do conceito da integralidade ao contexto do trabalho de enfermagem.

\section{RESULTADOS E DISCUSSÃO}

Considera-se que a alta da criança hospitalizada não significa uma completa recuperação da saúde, sendo assim, é necessária uma continuidade do cuidado após a alta hospitalar para um completo restabelecimento da criança. Nessa perspectiva, a fragmentação do cuidado reflete diretamente na qualidade da assistência, podendo resultar em reinternação.

Diante disso, identificamos no processo de trabalho de enfermagem na alta da criança hospitalizada que os enfermeiros deparam-se em seu dia-a-dia com algumas dificuldades que impedem e/ou limitam o cuidado integral à criança; por outro lado também existem potencialidades que contribuem para tal construção.

\section{Prática integral do enfermeiro: limites}

No que se refere aos limites na construção da integralidade do cuidado e sua continuidade na articulação entre atenção hospitalar e básica na pós-alta da criança hospitalizada, os entrevistados ressaltaram "lacunas no agir" e "lacunas no saber".

Como "lacunas no agir", um dos fatores mais citados nas entrevistas como limitador da atuação integral do enfermeiro é a "ausência de comunicação" entre a atenção hospitalar e a básica na pós-alta da criança. Os enfermeiros da atenção hospitalar e da básica reconhecem a inexistência de uma ponte entre os dois serviços de saúde, de uma ligação efetiva do processo de trabalho em saúde e, em foco neste estudo, da enfermagem: limite seria a falta de comunicação, não ter [...] um fluxo de como deveria ser esse atendimento, [...] como deveria ser articulado esta referência e contrarreferência (E3 ESF). Essa lacuna reflete uma fragmentação do cuidado e gera uma fragilidade na assistência integral no processo de alta hospitalar.

Ficou identificado que no processo de alta hospitalar não existe um protocolo para uma comunicação direta, que articule a atenção hospitalar e a básica. Em alguns casos específicos, as enfermeiras da atenção hospitalar realizam contato com os enfermeiros da atenção básica para repasse de informações sobre a criança em processo de alta. Já os enfermeiros da atenção básica afirmam, em quase sua totalidade, não haver essa articulação.

Para prestar a atenção integral, faz-se necessário a efetivação de uma rede integrada de serviços de saúde, diferente dos serviços fragmentados atuais. Sugere-se o desenvolvimento de formas de comunicação e negociação, como incentivo aos profissionais de saúde a participarem na integração dos serviços, possibilitando a formação de novos espaços de articulação das ações e dos saberes. ${ }^{20}$ 
Os próprios entrevistados levantaram algumas questões que poderiam estar gerando essa lacuna no cuidado, como: [...] desconhecer esses processos, [...] esquecer [...], de fazer esta contrarreferência [...] (E1 ESF); [...] não ter essa cultura de integralidade hospitalar e atenção básica [...] (E2 UIP).

Despertados para essa problemática, alguns dos entrevistados sugerem e acreditam na necessidade de um protocolo do processo de alta hospitalar, para que ocorra essa prática integral e consequentemente uma mudança no atual cenário: [...] se tivesse um protocolo do hospital ou da atenção básica, aos poucos a gente conseguiria mudar essa realidade (E2 UIP).

Os protocolos são apoios e direção para a ação do trabalho em saúde, porém não são suficientes ou capazes de abarcar toda a sua complexidade e dinamicidade, pois "as situações que emergem no cotidiano são sempre singulares, em condições específicas, com usuários diferentes e desdobramentos diversos". ${ }^{21: 20}$

Contudo, um profissional de enfermagem da atenção básica lembra que, antes de se estabelecer um protocolo, deve-se pensar nas condições de trabalho das equipes de ESF, especialmente quando algumas delas possuem um número reduzido de profissionais de saúde.

Assim, evidenciou-se uma deficiência na assistência com a falta de estratégias para essa parcela da população em processo de alta hospitalar: [...] efetivamente [...] não tem ainda uma estratégia de busca oficial nas unidades de internações hospitalares das crianças que estão em processo de alta, ou [...] internadas, para promover uma maior vigilância (E6 ESF).

As orientações realizadas na atenção hospitalar à criança dificilmente chegam às ULSs, gerando assim uma fragmentação no cuidado. Somente uma pequena parcela dos enfermeiros da atenção básica obtém essa informação através das próprias famílias que procuram a ULS logo após a alta, orientados pelas enfermeiras da atenção hospitalar.

Outro ponto sensível nesse agir é a falha na informação acerca do processo de alta hospitalar. Nesse aspecto, os enfermeiros da atenção básica relataram que não ficam cientes da maioria dos casos, ou reconhecem tardiamente as internações e o processo de alta hospitalar da criança em suas áreas de abrangência. Algumas vezes, essa informação é obtida algum tempo após a internação, seja pelos familiares das crianças ou pelo Agente Comunitário de Saúde (ACS). Isso aponta para uma deficiência na vigilância em saúde e contradiz o que é preco- nizado pela ESF, ao propor que a equipe esteja em condições de realizar um trabalho de vigilância.

Já as enfermeiras da atenção hospitalar relataram que, geralmente, obtém a informação de alta pelo médico responsável pela internação da criança, contudo, em alguns casos, esta só é identificada tardiamente, informada pelo próprio acompanhante da criança. Isso denota uma falha no trabalho em equipe e na própria concepção acerca do processo de alta, ainda tomado como alta médica, limitando as ações de enfermagem, que deveriam ocorrer ao longo da internação.

O acolhimento, outra atividade que pertence ao enfermeiro, foi citado também como fator limitante à prática integral do atendimento à saúde da criança. Essa prática em si, sabemos, contribui para a integralidade do cuidado, pois através dele também são "resgatadas" crianças em processo de alta hospitalar. Porém, nesse caso, o limite está associado ao comprometimento da maior parte da agenda desse profissional com essa prática, dificultando, assim, a assistência do enfermeiro à saúde da criança. Isso nos remete à necessidade de discutir a organização do trabalho da equipe em ambos os contextos, pois novas estratégias e metodologias podem perder seu potencial inovador quando se tornam apenas mais uma tarefa a ser cumprida, mais uma ação estanque, divorciada de seu sentido ou finalidade. Sem um projeto assistencial que articule ações e sujeitos, proposições metodológicas serão apenas um novo item no "pacote", algumas vezes implicando no abandono de outras funções. Embora esta perspectiva multiprofissional não tenha sido foco deste estudo, cabe reconhecer a necessidade de seu aprofundamento

Na percepção de parte dos entrevistados, a falta de tempo, associada às muitas funções que os enfermeiros da atenção hospitalar e da básica exercem em seus locais de trabalho, impossibilita a prática da articulação desses profissionais no processo de alta hospitalar: [...] limites têm bastante, excesso de trabalho, em alguns momentos o tempo que nós temos [...] (E1 UIP); [...] têm limites de sobrecarga de funções (E6 PSF). Apesar disso, alguns enfermeiros colocam que seria possível realizar um cuidado integral em uma dimensão sistêmica, numa prática que articule atenção hospitalar e básica no processo de alta da criança hospitalizada.

Esses dados aproximam-se dos que foram encontrados em outro estudo com equipes, que aponta a sobrecarga de trabalho, juntamente com a falta de tempo, como fatores que podem dificultar a articulação das ações na equipe. ${ }^{22}$ 
O déficit de recursos humanos é outro fator que aparece como limitador no processo de trabalho do enfermeiro da atenção básica, agregado às múltiplas funções desse profissional nas ULSs. Esse déficit muitas vezes impede o enfermeiro de realizar suas funções, entre elas a visita domiciliar, delegando-a ao ACS: [...] eu peço para o ACS ver como tá a criança [...] saber por que que ela internou, [...] sempre que eu posso, eu vou fazer visita [...] mas às vezes falta pessoal da enfermagem, [...] aí não consigo ir (E5 ESF).

Um estudo realizado em Porto Alegre desvelou dados semelhantes, mostrando que os enfermeiros deixam de realizar atividades próprias de sua competência profissional para cobrir atividades básicas de enfermagem nas ULSs. Coloca que a visita domiciliar é uma das atividades limitadas pela falta do profissional enfermeiro. ${ }^{23}$

Outro fator limitante da prática integral, citado pelo enfermeiro da atenção hospitalar, seria a desarticulação dos prontuários informatizados existentes na atenção hospitalar e na básica, que não são integrados, o que desfavorece a comunicação entre esses sistemas.

Em relação às "lacunas no saber", foram apontados os seguintes fatores limitantes para a construção da atenção integral e articulação do trabalho de enfermagem no processo de alta hospitalar: a falta de conhecimento acerca do cuidado integral, a "falha" na formação desses profissionais e na continuidade do aprendizado durante sua trajetória profissional. A formação acadêmica não-reducionista e menos fragmentada seria fundamental para uma assistência mais integral. ${ }^{4}$

O déficit na formação do enfermeiro na questão da consulta à criança foi outro fator apontado como limitante do trabalho de enfermagem na atenção básica. Alguns profissionais não se sentem preparados para tal atividade: [...] tem muitos enfermeiros que [...] não se sentem aptos à própria puericultura [...] (E6 ESF); Como lidar com uma criança que não tem ainda uma saúde restabelecida completamente, como seguir dali pra frente? (E2 ESF). A ESF deve reorganizar a atenção básica, e necessita garantir, entre outras coisas, um adequado programa de educação permanente.

Em estudo realizado com enfermeiros de Goiânia sobre a integralidade da assistência, foram identificados, como fatores limitantes, a formação acadêmica, a falta de qualificação dos profissionais de saúde e o desinteresse em manterse atualizados. ${ }^{4}$
Prática integral do enfermeiro: potencialidades

Os enfermeiros colocam, além dos limites, potencialidades em seu processo de trabalho para a integralidade do cuidado na articulação da atenção hospitalar e da atenção básica/ESF na pós-alta da criança/família.

A alta cobertura das equipes de ESF no município de estudo e a reorganização do processo de trabalho em saúde foram as principais potencialidades identificadas nos relatos dos enfermeiros: [...] cada bairro tem seu centro de saúde, tem sua equipe de ESF (E4 UIP); [...] é que a atenção básica tá conseguindo dar conta da atenção da criança [...] (E2 UIP).

Nos relatos, os enfermeiros colocam que com a implantação das equipes de ESF evidenciou-se uma redução no número de internações na Unidade de Internação Pediátrica. Acredita-se que, caso houvesse uma articulação direta entre a atenção básica e a hospitalar na pós-alta da criança hospitalizada, o número de reinternações também seria menor.

Uma das mais citadas potencialidades foi o perfil do enfermeiro. Percebemos nas falas que a prática integral está relacionada com a atuação individual, com a forma de cada profissional atuar nas necessidades de cada criança/família em processo de alta, na perspectiva da integralidade do cuidado: uma delas é a enfermeira ser bem dinâmica, ela ir atrás [...], dar um jeito [...] pra que ela vá bem de alta pra casa [...] (E1 UIP). A prática da integralidade está vinculada diretamente ao perfil do profissional membro da equipe de saúde. ${ }^{24}$

$O$ trabalho em equipe interfere nessa configuração e foi reconhecido como outra potencialidade. Neste município, novas equipes estão sendo formadas e outras equipes estão recebendo novos profissionais efetivos, promovendo uma reorganização do trabalho: [...] tanto a nível de unidade hospitalar quanto de atenção básica, eu acho que a gente tem equipes de saúde bem fortalecidas (E2 UIP). O agir com o outro se faz necessário para promover um cuidado integral, à medida que cada profissional necessita do outro para dar continuidade a esse cuidado. A continuidade do cuidado é um dos elementos estruturais necessários para construir a integralidade ${ }^{8}$, que presume um encontro entre práticas e saberes efetivos de diversos profissionais. ${ }^{25}$ Quando os diferentes profissionais realizam trocas de informações, conhecimentos e decisões em equipe, aí acontece 
a articulação das ações. ${ }^{22}$ Embora a integralidade remeta a esta perspectiva multiprofissional, não significa que não possa e deva ser explorada em relação às práticas e desafios de cada profissional, como no caso deste estudo, que a aborda do ponto de vista da enfermagem.

Apesar dos obstáculos para atingir o destino final, as orientações dos enfermeiros da atenção hospitalar à criança/família em processo de alta foram identificadas como potencializadoras da articulação com a atenção básica, uma ferramenta para a construção do cuidado integral, apesar de nem sempre chegar ao seu destino final: [...] ele veio bem orientado (E4 ESF); [...] a mãe saiu da alta e veio pra unidade por orientação do hospital pra marcar uma consulta de acompanhamento mais cedo (E2 ESF).

A capacitação, como potencialidade, foi identificada pela busca dos profissionais da atenção hospitalar pelo estudo e pelo fato de profissionais ligados a um hospital escola se perceberem mais preparados e motivados para desenvolverem uma prática efetiva na articulação do trabalho no processo de alta da criança: [...] a vontade que a gente tem, por ser um hospital escola, [...] estar sempre estudando, todas nós estamos nos reciclando [...]. Cada um que vai buscar uma universidade ou um mestrado, um doutorado, estar trazendo novas expectativas pra gente, novas esperanças de melhorar nossa assistência (E6 UIP).

Por fim, a sistematização da assistência de enfermagem utilizada no hospital pesquisado e o programa da atenção básica nomeado Capital Criança - que prevê uma atenção especial à criança - foram considerados como potencialidades, ferramentas para a articulação no processo de alta hospitalar da criança e, consequentemente, para a continuidade do cuidado.

\section{Contradições: prática integral do enfermeiro - limites ou potencialidades?}

Considerou-se importante também ressaltar relatos que demonstraram certas contradições, nos quais atores integrantes do trabalho da atenção básica despontam como limitantes e também potencializadores desse processo. São eles: o pediatra e o ACS.

Na atenção básica, o pediatra foi identificado como limitante na maioria dos relatos, porque assiste todas as crianças que procuram as ULSs, ou a maior parte dessa demanda. Desvelou-se que são poucas as equipes de ESF em que a proposta do matriciamento em pediatria funciona em conformidade com o que é preconizado pelo Ministério da Saúde. Esse profissional deveria ser apoio à equipe, porém, no cotidiano da atenção à saúde da criança, a presença do pediatra foi vista como obstáculo ao processo de trabalho de enfermagem nessa área, já que dificilmente essa população chega às consultas de enfermagem: [...] aqui a gente não tem contato com as crianças. Por essa presença do especialista dentro da unidade, a gente não trabalha como equipe de saúde da família no atendimento integral à criança [...] (E3 ESF).

Uma pequena parcela dos relatos identificou o pediatra como potencialidade, quando esses profissionais compartilham saberes e práticas com o enfermeiro e conseguem trabalhar em equipe. Ressaltou-se a importância da consulta de enfermagem à saúde da criança, bem como a consulta médica, ambas distintas, cada qual com sua importância, sendo que juntas favorecem a consolidação doSUS e de seus princípios. Considera-se a consulta de enfermagem um instrumento que concede ao enfermeiro uma prática específica de seu fazer, bem como um instrumento metodológico e tecnológico para o cuidar, ${ }^{26}$ contudo, não pode isoladamente contemplar a assistência à saúde da criança.

A atuação do ACS também foi citada, tanto como limite quanto como potencialidade no processo de alta hospitalar. Na maioria dos relatos, os enfermeiros afirmam que nem sempre os ACSs repassam a informação acerca da alta hospitalar: [...] como limite às vezes o próprio ACS (E2 ESF). Entre outras atribuições, cabe aos ACSs acompanharem mensalmente as famílias de sua área de atuação, realizar ações básicas de saúde à criança e informar a equipe de saúde sobre as necessidades da comunidade, inclusive os casos de internações. Aos enfermeiros da ESF cabe, entre outras atribuições, assistir a criança, capacitar os ACSs e organizar o trabalho das ULSs. Reforçando a compreensão deste papel, o ACS também é visto como potencialidade, ainda que em menor proporção, quando ele consegue detectar a criança/ família em processo de alta hospitalar e repassar essa informação ao enfermeiro coordenador da equipe: os ACs são bem atuantes (E5 ESF); [...] têm os ACs, isso são potencialidades [...] (E3 ESF).

Dessa forma, acredita-se que o pleno desenvolvimento das atribuições previstas por cada integrante da equipe é elemento basal para que a ESF configure-se em sua potencialidade na construção do SUS e de seus princípios, incluída a integralidade, aqui retomada como o foi ao longo do estudo, em seus múltiplos sentidos, especialmente como ferramenta para organizar as práti- 
cas, atuando no nível de políticas, dos serviços e das práticas. Especialmente, considerando que a compreensão sobre práticas particulares, como de enfermeiros no pós-alta da criança, apesar de seus limites, têm relevância para pensar a integralidade sob o foco da organização dos serviços pois não acontecem fora dele e apontam para desafios a serem superados neste nível. Ou seja, estes três níveis não são desvinculados e o sucesso de uma política que elege como princípio a integralidade dependerá de sua operacionalização em múltiplas e particulares ações.

Portanto, a organização do trabalho de enfermagem implica nas relações dos profissionais de enfermagem entre si e com outros profissionais e usuários do serviço de saúde; nas facilidades e fragilidades provocadas pela estrutura organizacional; na divisão de trabalho; e nas relações estabelecidas com as demais instituições que fazem parte do sistema de saúde. ${ }^{27}$

\section{CONSIDERAÇÕES FINAIS}

Os resultados da pesquisa apontam que no cotidiano de trabalho, na alta da criança hospitalizada, em busca de uma prática integral, os enfermeiros encontram alguns limites e potencialidades que dificultam ou favorecem um agir coerente com o princípio da integralidade.

No que se refere aos limites, evidenciaram-se lacunas no agir e lacunas no saber. Na primeira, despontam questões relacionadas à organização do trabalho de enfermagem no processo de alta hospitalar da criança/família. Nesta, ressalta-se a falta de comunicação entre os sistemas, caracterizando a desarticulação entre eles. Na segunda, despontam questões como a "falha" na formação e na educação permanente.

Quanto às potencialidades, foram citadas questões individuais, como o perfil do enfermeiro, e questões coletivas, como o trabalho em equipe e o potencial da alta cobertura das ESFs no município em que ocorreu a pesquisa e seu reflexo na redução de internações da criança/família.

Apesar de claras indicações de elementos ou circunstâncias do trabalho que favorecem ou prejudicam a articulação da atenção básica com a hospitalar, contradições também são reveladoras de certas dificuldades do trabalho multiprofissional. Isso se mostra, especialmente, quando alguns atores desse processo, como o pediatra e o ACS, aparecem tanto como limites quanto como potencialidades.
Assim, considera-se que este estudo, ao reconhecer os limites e as potencialidades da prática integral do enfermeiro no processo de alta hospitalar da criança, favorece um enfrentamento dessa problemática pelos profissionais envolvidos, de modo a contemplar melhor a articulação entre atenção hospitalar e básica.

Conclui-se que, na perspectiva da integralidade, faz-se necessário um refletir, um repensar dos profissionais, na busca por caminhos e estratégias que possam modificar o atual cenário e preencher as lacunas existentes no cuidado prestado e na articulação entre seus diferentes níveis. Acreditase que preencher essas lacunas é sem dúvida um desafio para os enfermeiros, porém, necessário para que mudanças ocorram na reorganização do trabalho, tendo em vista a promoção da saúde da criança e a redução de reinternações.

\section{REFERÊNCIAS}

1. Vasconcelos CM, Pasche DF. O Sistema Único de Saúde. In: Campos GWS, Minayo MCS, Akerman M, Drumond M Junior, Carvalho YM, organizadores. Tratado de saúde coletiva. São Paulo/Rio de Janeiro (SP/RJ): Hucitec/Fiocruz; 2006. p. 531-62.

2. Brasil. Lei n. 8080, de 19 de setembro de 1990: dispõe sobre as condições para a promoção, proteção e recuperação da saúde, a organização e o funcionamento dos serviços correspondentes e dá outras providências. Brasília (DF): Guia do Conselheiro; 2002.

3. Fontoura RT, Mayer CN. Uma breve reflexão sobre a integralidade. Rev Bras Enferm. 2006 Jul-Ago; 59(4):532-6.

4. Pinho IC, Siqueira JCBA, Pinho LMO. As percepções do enfermeiro acerca da integralidade da assistência. Rev Eletr Enferm [online]. 2006 [acesso 2009 Jul 06]; 8(1):42-51. Disponível em: http:/ / www.fen.ufg.br/ revista/revista8_1/original_05.htm.

5. Mattos RA. Integralidade e a formulação de políticas específicas de saúde. In: Pinheiro R, Mattos RA, organizadores. Construção da integralidade: cotidiano, saberes e práticas em saúde. $3^{\mathrm{a}}$ ed. Rio de Janeiro (RJ): IMS/UERJ/ Abrasco; 2005. p. 45-59.

6. Silva VC. O processo de implantação do sistema integrado de serviços de saúde em Vitória - ES; contribuição à discussão da integralidade na atenção à saúde [dissertação]. Rio de Janeiro (RJ): Escola Nacional de Saúde Pública; 2004.

7. Cecílio LCO, Merhy EE. A integralidade do cuidado como eixo da gestão hospitalar. In: Pinheiro R, Mattos RA, organizadores. Construção da integralidade: cotidiano, saberes e práticas em saúde. $3^{\mathrm{a}}$ ed. Rio de Janeiro (RJ): IMS, UERJ, Abrasco; 2005. p. 197-210. 
8. Starfield B. Atenção primária: equilíbrio entre necessidades de saúde, serviços e tecnologias. Brasília (DF): UNESCO, Ministério da Saúde; 2002.

9. Issi HB, Schenkel SS, Lautuada VTV. Hospitalização da criança e participação da família. Programa de Atualização em Enfermagem: saúde da criança e do adolescente: PROENF - ciclo 2, módulo 2. Porto Alegre (RS): Artmed; 2007.

10. Pimenta EAG, Collet N. Dimensão cuidadora da enfermagem e da família na assistência à criança hospitalizada: concepções da enfermagem. Rev Esc Enferm USP. 2009 Set; 43(3):622-9.

11. Schatkoski AM, Wegner W, Algeri S, Pedro ENR. Segurança e proteção à criança hospitalizada: revisão de literatura. Rev Latino-am Enferm. 2009 Mai-Jun; 17(3):410-6.

12. Ceribelli C, Nascimento LC, Pacifico SMR, Lima RAG. A mediação de leitura como recurso de comunicação com crianças hospitalizadas. Rev Latino-am Enferm. 2009 Jan-Fev; 17(1):81-7.

13. Pinheiro EM, Silva MJP, Margareth A, Ribeiro CA. A importância da interação do enfermeiro com recém-nascido/família durante a internação. Rev Latino-am Enferm. 2008 Nov-Dez; 16(6):1012-8.

14. Mello DF, Lima RAG, Scochi CGS. A saúde de crianças em situação de pobreza: entre a rotina e a eventualidade de cuidados cotidianos. Rev Latinoam Enferm. 2007 Set-Out; 16(Esp):820-7.

15. Matumoto S, Mishima SM, Fortuna CM, Pereira MJB, Almeida MCP. Preparando a relação de atendimento: ferramenta para o acolhimento em unidades de saúde. Rev Latino-am. Enferm 2009 Nov-Dez; 17(6):1001-8.

16. Shimizu HE, Rosales C. A atenção básica da família sob a ótica do usuário. Rev Latino-am. Enferm. 2008 Set-Out; 16(5):883-8.

17. Figueiredo GLA, Mello DF. Atenção à saúde da criança no Brasil: aspectos da vulnerabilidade programática e dos direitos humanos. Rev Latinoam Enferm. 2007 Nov-Dez; 15(6):1171-6.

18. Couto FC, Praça NS. Preparo dos pais de recémnascido prematuro para alta hospitalar: uma revisão bibliográfica. Esc Anna Nery Rev Enferm 2009 OutDez; 13(4):886-91.

19. Dyniewicz AM. Metodologia da pesquisa em saúde para iniciantes. São Caetano do Sul (SP): Difusão Editora; 2007.
20. Costa Silva V, Rivera FJU, Hortale VA. Projeto Integrar: avaliação da implantação de serviços integrados de saúde no Município de Vitória, Espírito Santo, Brasil. Cad Saúde Pública. 2007 Jun; 23(6):1405-14.

21. Gomes RS, Silva FH, Pinheiro R, Barros MEB. Integralidade como princípio ético e formativo: um ensaio sobre valores éticos para estudos sobre o trabalho em equipe na saúde. In: Pinheiro R, Barros MEB, Mattos RA, organizadoras. Trabalho em equipe sob o eixo da integralidade: valores, saberes e práticas. Rio de Janeiro (RJ): CEPESC; 2007. p. 19-36.

22. Colomé ICS, Lima MADS, Davis R. Visão de enfermeiras sobre as articulações das ações de saúde entre profissionais de equipes de saúde da família. Rev Esc Enferm USP. 2008 Jun; 42(2):256-61.

23. Nauderer TM, Lima MADS. Práticas de enfermeiros em unidades básicas de saúde em Município do Sul do Brasil. Rev Latino-am Enferm. 2008 Set-Out; 16(5):889-94.

24. Mattos RA. Os sentidos da integralidade: algumas reflexões acerca de valores que merecem ser defendidos. In: Pinheiro R, Mattos RA, organizadores. Os sentidos da integralidade na atenção e no cuidado à saúde. Rio de Janeiro (RJ): UERJ/IMS/ABRASCO; 2001. p. 39-63.

25. Louzada APF, Bonaldi CB, Barros MEB. Integralidade e trabalho em equipe no campo da saúde: entre normas antecedentes e recentradas. In: Pinheiro R, Barros MEB, Mattos RA, organizadores. Trabalho em equipe sob o eixo da integralidade: valores, saberes e práticas Rio de Janeiro (RJ): CEPESC; 2007. p. 37-52.

26. Brasil. Portaria n. 154 GM, de 24 de janeiro de 2008: Cria os núcleos de apoio à saúde da família - NASF. [online] [acesso 2009 Ago 14]. Disponível em: www. saude.gov.br/portal

27. Santos I, Correa LA, Albuquerque DC. A consulta de enfermagem através da escuta sensível e diagnósticos para o autocuidado do cliente com insuficiência cardíaca. In: Santos I, David HMSL, Silva D, Melo CM, organizadores. Enfermagem e campos de prática em saúde coletiva: realidade, questões e soluções. São Paulo (SP): Ateneu; 2008. p.271-280.

28. Matos E, Pires DEP. A organização do trabalho da enfermagem na perspectiva dos trabalhadores de um hospital escola. Texto Contexto Enferm. 2002 Jan-Mar; 11(1):187-205. 